\title{
Differential distributions for top-quark hadro-production with a running mass
}

\author{
M. Dowling ${ }^{1, a}$, S. Moch $^{1,2}$ \\ ${ }^{1}$ Deutsches Elektronensynchrotron DESY, Platanenallee 6, 15738 Zeuthen, Germany \\ 2 II. Institut für Theoretische Physik, Universität Hamburg, Luruper Chaussee 149, 22761 Hamburg, Germany
}

Received: 21 October 2013 / Accepted: 31 October 2014 / Published online: 18 November 2014

(C) The Author(s) 2014. This article is published with open access at Springerlink.com

\begin{abstract}
We take a look at how the differential distributions for top-quark production are affected by changing to the running mass scheme. Specifically we consider the transverse momentum, rapidity and pair-invariant mass distributions at next-to-leading order for the top-quark mass in the $\overline{\mathrm{MS}}$ scheme. It is found that, similar to the total cross section, the perturbative expansion converges faster and the scale dependence improves using the mass in the $\overline{\mathrm{MS}}$ scheme as opposed to the on-shell scheme. We also update the analysis for the total cross section using the now available full next-to-next-to-leading order contribution.
\end{abstract}

The measurement of top-quark pair production cross sections at hadron colliders has entered the era of precision physics with the analysis of data available from the Large Hadron Collider (LHC) in the runs at center-of-mass energies $\sqrt{S}=7$ and $8 \mathrm{TeV}$. Measurements of the total cross section for $t \bar{t}$-production from ATLAS and CMS reach by now an accuracy of typically better than $\mathscr{O}(10 \%)$, with the systematic and luminosity uncertainties already dominating over the small statistical uncertainty, see, e.g., [1-3]. First results of differential distributions for $t \bar{t}$-production from the LHC are appearing as well $[4,5]$. Thus, given the present experimental accuracy hadro-production of $t \bar{t}$-pairs is currently being established as a standard model (SM) benchmark process.

This has motivated tremendous activity on the theory side to match the experimental precision by computing higher order corrections in quantum chromodynamics (QCD) and we briefly recapitulate the status for inclusive $t \bar{t}$-pair production, i.e., no tagged final states. Predictions for the total cross section are complete to next-to-next-to-leading order (NNLO) [6-9] while differential distributions are known to next-to-leading order (NLO) $[10,11]$, including top-quark decay [12-16], though. Additional corrections beyond NLO based on threshold logarithms have been obtained for distri-

\footnotetext{
a e-mail: matthew.dowling@desy.de
}

butions in the top-quark's transverse momentum and rapidity, $p_{\mathrm{T}}^{t}$ and $y^{t}$, as well as in the invariant mass $m^{t \bar{t}}$ of the top-quark pair [17-20].

Comparison of these theory predictions to experimental data can be used to determine parameters such as the strong coupling constant, the parton luminosity and the top-quark mass and to study their correlations. Of these parameters, the top-quark mass is certainly the most interesting one with prominent implications for the electro-weak vacuum of the $\mathrm{SM}$, see, e.g., [21,22]. It is a particularly attractive feature of cross sections measurements that they offer the opportunity for an unambiguous and theoretically well-defined determination of the top-quark mass in a particular renormalization scheme $[23,24]$.

The conventional scheme choice for the quark mass renormalization is the pole mass, which has its short-comings [25, 26], though, since it is based on the idea of quarks appearing as asymptotic states. It exhibits poor convergence of the perturbative series and due to the renormalon ambiguity it carries an intrinsic uncertainty of the order of $\Lambda_{\mathrm{QCD}}$. As an alternative, one can consider top-quark hadro-production with a running mass, which has the advantages of improved convergence and scale stability of the perturbative expansion. For $t \bar{t}$ hadro-production, these features have been demonstrated for the total cross section [23].

In the present letter, we study the dependence of single differential distributions in $p_{\mathrm{T}}^{t}, y^{t}$ and $m^{t \bar{t}}$ on the definition of the mass parameter. Specifically, we will compare the conventional pole mass $m_{t}^{\text {pole }}$ with the scale dependent $\overline{\mathrm{MS}}$ mass by means of the well-known relation in perturbation theory,

$m_{t}^{\text {pole }}=m\left(\mu_{\mathrm{r}}\right)\left(1+\frac{\alpha_{\mathrm{s}}}{\pi} d_{1}+\left(\frac{\alpha_{\mathrm{s}}}{\pi}\right)^{2} d_{2}+\cdots\right)$,

for the scheme change from $m_{t}^{\text {pole }}$ to the running $\overline{\mathrm{MS}}$ mass $m\left(\mu_{\mathrm{r}}\right)$ taken at the renormalization scale $\mu_{\mathrm{r}}$. To NNLO the coefficients $d_{1}$ and $d_{2}$ are given by [27] (see also [28,29]) 


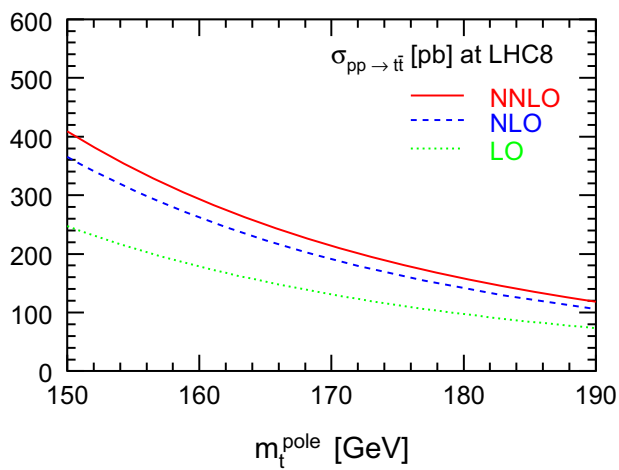

Fig. 1 The LO, NLO and NNLO QCD predictions for the total cross section at $\mathrm{LHC}(\sqrt{S}=8 \mathrm{TeV})$ as a function of the top-quark mass in the on-shell scheme $m_{t}^{\text {pole }}$ at the scale $\mu=m_{t}^{\text {pole }}$ (left) and, respectively, in

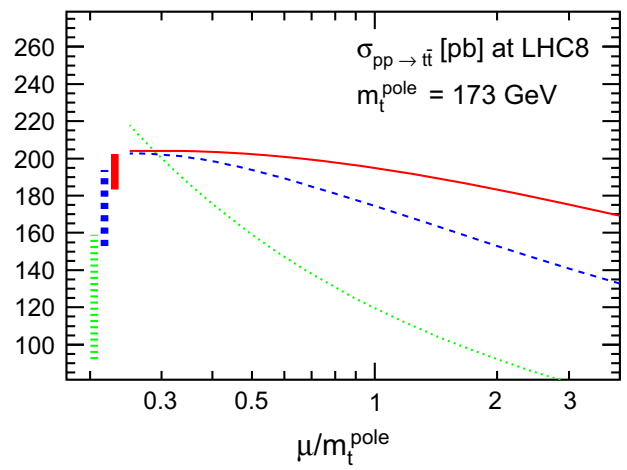

Fig. 2 The scale dependence of the LO, NLO and NNLO QCD predictions for the total cross section at $\operatorname{LHC}(\sqrt{S}=8 \mathrm{TeV})$ for the top-quark mass $m_{t}^{\text {pole }}=173 \mathrm{GeV}$ in the on-shell scheme (left) and for $m(m)=163 \mathrm{GeV}$ in the $\overline{\mathrm{MS}}$ scheme (right) with the choice

$$
\begin{aligned}
d_{1}= & \frac{4}{3}+\ell \\
d_{2}= & \frac{307}{32}+\frac{\pi^{2}}{3}+\frac{\pi^{2}}{9} \ln (2)-\frac{1}{6} \zeta_{3}+\frac{509}{72} \ell+\frac{47}{24} \ell^{2} \\
& -n_{\mathrm{f}}\left(\frac{71}{144}+\frac{\pi^{2}}{18}+\frac{13}{36} \ell+\frac{1}{12} \ell^{2}\right),
\end{aligned}
$$

with $\ell=\ln \left(\frac{\mu_{\mathrm{r}}^{2}}{m\left(\mu_{\mathrm{r}}\right)^{2}}\right)$ and assuming vanishing masses for all lighter quarks.

Let us briefly illustrate the advantages of the running $\overline{\mathrm{MS}}$ mass $m\left(\mu_{\mathrm{r}}\right)$ for the total $t \bar{t}$ cross section. The recently completed exact NNLO QCD result [6-9] turned out to provide a value for the total cross section which is very close, i.e., within $\mathscr{O}(1-5 \%)$, to previous approximations based on the combined threshold and high-energy asymptotics [31] and has been presented as a function of the pole mass $m_{t}^{\text {pole }}$. The necessary scheme transformation from $m_{t}^{\text {pole }}$ to $m\left(\mu_{\mathrm{r}}\right)$, i.e., the application of Eq. (1), has been discussed in [23] and is implemented in the program Hathor (version 1.5) [32], a

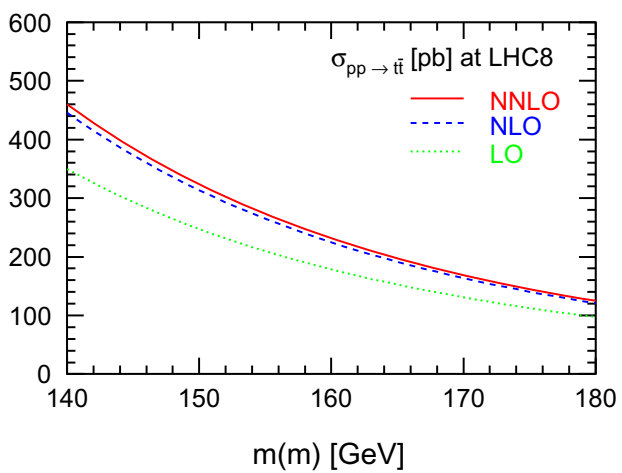

the $\overline{\mathrm{MS}}$ scheme $m(m)$ at the scale $\mu=m(m)$ (right) using the PDF set ABM11 [30] and $\mu=\mu_{\mathrm{r}}=\mu_{\mathrm{f}}$

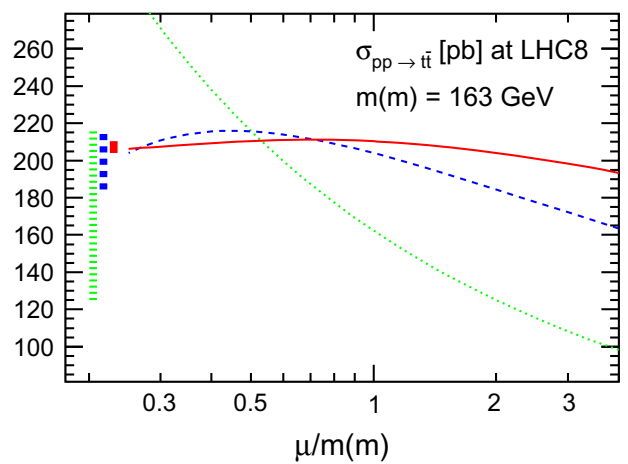

$\mu=\mu_{\mathrm{r}}=\mu_{\mathrm{f}}$ using the PDF set ABM11 [30]. The vertical bars indicate the size of the scale variation in the standard range $\mu / m_{t}^{\text {pole }} \in[1 / 2,2]$ and $\mu / m(m) \in[1 / 2,2]$, respectively

tool for the calculation of the total $t \bar{t}$ cross section in hadronic collisions.

The much improved apparent convergence of the perturbative expansion with the running mass as well as the scale stability are illustrated in Figs. 1 and 2 where we compare theory predictions for the total $t \bar{t}$ cross section as a function of the pole and the $\overline{\mathrm{MS}}$ mass, respectively. Figure 1 displays the increase in the cross section values from LO to NNLO, where we have taken the parton distribution functions (PDFs) to be order independent. For an on-shell mass $m_{t}^{\text {pole }}=173 \mathrm{GeV}$, for instance, the relative increase is $\sigma_{\mathrm{NLO}} / \sigma_{\mathrm{LO}}=1.46$ and $\sigma_{\mathrm{NNLO}} / \sigma_{\mathrm{NLO}}=1.12$ at the scale $\mu_{\mathrm{r}}=\mu_{\mathrm{f}}=m_{t}^{\text {pole }}$. This is to be compared with a much reduced increase of only $\sigma_{\mathrm{NLO}} / \sigma_{\mathrm{LO}}=1.26$ and $\sigma_{\mathrm{NNLO}} / \sigma_{\mathrm{NLO}}=1.03$ for $m(m)=163 \mathrm{GeV}$ in the $\overline{\mathrm{MS}}$ scheme at the scale $\mu_{\mathrm{r}}=\mu_{\mathrm{f}}=m(m)$. These findings can be understood by noting that the scheme transformation of Eq. (1) applied to the total $t \bar{t}$ cross section effectively shifts all corrections of the Wilson coefficients of the perturbative cross-section to the threshold region thereby improving the 


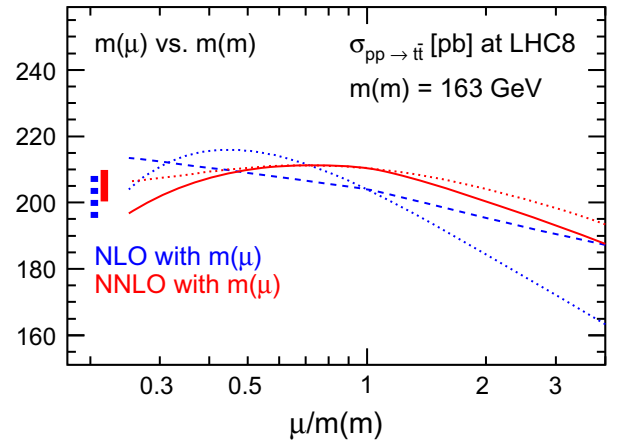

Fig. 3 The scale dependence of the total cross section at LHC $(\sqrt{S}=$ $8 \mathrm{TeV}$ ) as a function of $m(\mu)$ in the $\overline{\mathrm{MS}}$ scheme for the NLO (blue, dashed) and NNLO (red, solid) QCD predictions with the choice $\mu=$ $\mu_{\mathrm{r}}=\mu_{\mathrm{f}}$ and using the PDF set ABM11 [30]. The scale variation is normalized to $m(m)=163 \mathrm{GeV}$. The vertical bars indicate the size of the scale variation in the standard range $\mu / m_{t}^{\text {pole }} \in[1 / 2,2]$ and $\mu / m(m) \in[1 / 2,2]$, respectively. The dotted curves display the results for the mass at the fixed scale $m(m)=163 \mathrm{GeV}$ as shown in Fig. 2 (right) for comparison

apparent convergence of the perturbation series, see, e.g., [33].

Figure 2 shows the scale stability for the LHC predictions confirming earlier findings for the Tevatron, cf. [23]. The scale variation for the cross section in the on-shell scheme in the standard range $\mu / m_{t}^{\text {pole }} \in[1 / 2,2]$ amounts to $\Delta \sigma_{\mathrm{NNLO}}={ }_{-6.0 \%}^{+3.8 \%}$, whereas for the running mass we only find $\Delta \sigma_{\mathrm{NNLO}}={ }_{-3.0}^{+0.1 \%}$ for the range $\mu / m(m) \in[1 / 2,2]$. Interestingly, for an on-shell mass the point of minimal sensitivity where $\sigma_{\mathrm{LO}} \simeq \sigma_{\mathrm{NLO}} \simeq \sigma_{\mathrm{NNLO}}$ is located at fairly low scales, $\mu \simeq m_{t}^{\text {pole }} / 4 \simeq 45 \mathrm{GeV}$, whereas for a running mass it resides at the scale $\mu=\mathscr{O}(m(m))$, i.e., it coincides with the natural hard scale of the process. The observation of improved scale stability for predictions with the $\overline{\mathrm{MS}}$ mass is further corroborated by studying alternative scale choices for $m(\mu)$ as shown in Fig. 3. Cross section predictions with the running mass $m(\mu)$ display a comparable uncertainty of $\Delta \sigma_{\mathrm{NNLO}}={ }_{-4.8 \%}^{+0.0 \%}$ for the range $81.5 \mathrm{GeV} \leq \mu \leq 326 \mathrm{GeV}$,

Table 1 The total cross section for top-quark pair-production at NNLO using a pole mass $m_{t}^{\text {pole }}=173 \mathrm{GeV}$ and the PDF set ABM11 [30] and CT10 [36] and with the errors shown as $\sigma+\Delta \sigma_{\text {scale }}+\Delta \sigma_{\mathrm{PDF}}$. The NLO numbers in brackets are obtained by using the respective NLO which corresponds to the range $\mu / m(m) \in[1 / 2,2]$ if normalized to $m(m)=163 \mathrm{GeV}$.

If instead, only the scale of the mass $m(\mu)$ is varied within $m(81.5 \mathrm{GeV}) \leq m(\mu) \leq m(326 \mathrm{GeV})$, while the scales $\mu_{\mathrm{r}}$ and $\mu_{\mathrm{f}}$ are kept fixed, one obtains shifts of $\Delta \sigma_{\mathrm{NNLO}}={ }_{-5.6 \%}^{+0.0 \%}$ relative to the cross section value at $m(m)$ for the choice $\mu_{\mathrm{r}}=$ $\mu_{\mathrm{f}}=163 \mathrm{GeV}$. Again, these variations are similar to the uncertainty quoted in Fig. 2 (right). These results imply, that experimental determinations of the running mass from the measured cross section are feasible with very good accuracy and a small residual theoretical uncertainty. For Tevatron data such analyses have already been performed in the past $[22$, 34]. Recently, the top-quark cross section data from Tevatron and LHC has been analyzed in a global fit at NNLO [35] with full account of all correlations between the top-quark mass, the gluon PDF and the strong coupling $\alpha_{\mathrm{s}}$.

For completeness, we include in Tables 1 and 2 the values for the full NNLO cross sections at the Tevatron $(\sqrt{S}=$ $1.96 \mathrm{TeV}$ ) and at the LHC for various energies of interest.

We also briefly comment here on a related study, that has appeared in the literature. Ahrens et al. [37] has performed a study of total $t \bar{t}$ cross section in the $\overline{\mathrm{MS}}$ scheme compared to the pole scheme for the top-quark mass and did not find any strong improvement of the perturbative series when using the running mass. This is in contradiction to the numbers reported in Tables 1 and 2. Note however, that the authors of [37] have used an approximation for the NNLO QCD corrections, which was appropriate at the time of writing, but has turned out to be inaccurate if compared to the exact NNLO QCD result [6-9]. In fact, [37] has predicted $t \bar{t}$ cross sections at approximate NNLO QCD accuracy, which are systematically too low by $\mathscr{O}(6-13 \%)$ depending on the collider and the center-of-mass energy.

Next we discuss the single-differential distributions in the top-quark's transverse momentum $p_{\mathrm{T}}^{t}$ and rapidity $y^{t}$ and in the invariant mass $m^{t \bar{t}}$ of the $t \bar{t}$-pair, which are all known to NLO in QCD $[10,11]$ in the conventional pole mass scheme. As we are interested in the differential cross sections with

PDF sets. The scale uncertainty $\Delta \sigma_{\text {scale }}$ is based on maximal and minimal shifts for the choices $\mu=m_{t}^{\text {pole }} / 2$ and $\mu=2 m_{t}^{\text {pole }}$ and $\Delta \sigma_{\mathrm{PDF}}$ is the $1 \sigma$ combined $\mathrm{PDF}+\alpha_{\mathrm{s}}$ error. All rates are in $\mathrm{pb}$

\begin{tabular}{|c|c|c|c|c|}
\hline & $\mathrm{TEV} \sqrt{S}=1.96 \mathrm{TeV}$ & $\mathrm{LHC} \sqrt{S}=7 \mathrm{TeV}$ & $\mathrm{LHC} \sqrt{S}=8 \mathrm{TeV}$ & $\mathrm{LHC} \sqrt{S}=14 \mathrm{TeV}$ \\
\hline \multirow[t]{2}{*}{ NLO: ABM11 } & $6.36_{-0.59}^{+0.21}{ }_{-0.15}^{+0.15}$ & $118.9_{-14.8}^{+13.2}+6.2$ & $174.5_{-21.4}^{+19.4}{ }_{-8.4}^{+8.4}$ & $738.8_{-83.8}^{+81.0}+24.8$ \\
\hline & $\left(6.50_{-0.64}^{+0.23+0.15}+{ }_{-0.15}^{+0.15}\right)$ & $\left(124.4_{-16.3}^{+14.7}+5.9\right)$ & $\left(182.4_{-23.5}^{+21.6}+8.0\right)$ & $\left(777.2_{-93.2}^{+91.5}{ }_{-25.1}^{+25.1}\right)$ \\
\hline \multirow[t]{2}{*}{ CT10 } & $6.72_{-0.73-0.32}^{+0.34}+0.43$ & $150.4_{-19.6-10.7}^{+18.0+13.3}$ & $215.5_{-27.5}^{+25.6+16.7}$ & $842.4_{-97.5}^{+98.0+33.5}$ \\
\hline & $\left(6.79_{-0.74}^{+0.34}+0.48\right)$ & $\left(149.7_{-19.4}^{+17.8+11.3}\right)$ & $\left(214.2_{-27.2}^{+25.4+16.3}+14.4\right)$ & $\left(839.5_{-97.5}^{+97.9+36.0}+36\right.$. \\
\hline NNLO: ABM11 & $6.82_{-0.29}^{+0.21}{ }_{-0.16}^{+0.16}$ & $133.0_{-8.2}^{+5.2}+6.5$ & $194.9_{-11.7}^{+7.4}{ }_{-8.8}^{+8.8}$ & $821.0_{-43.7-25.7}^{+27.0}+25.7$ \\
\hline CT10 & $7.30_{-0.39}^{+0.28+0.33}+0.45$ & $168.9_{-10.9}^{+6.9}{ }_{-10.9}^{+13.5}$ & $241.6_{-15.1}^{+9.5}+13.8$ & $939.3_{-51.7-33.3}^{+32.4}$ \\
\hline
\end{tabular}


Table 2 Same as Table 1 for a running mass $m(m)=163 \mathrm{GeV}$ in the $\overline{\mathrm{MS}}$ scheme

\begin{tabular}{|c|c|c|c|c|}
\hline & $\mathrm{TEV} \sqrt{S}=1.96 \mathrm{TeV}$ & $\mathrm{LHC} \sqrt{S}=7 \mathrm{TeV}$ & $\mathrm{LHC} \sqrt{S}=8 \mathrm{TeV}$ & $\overline{\mathrm{LHC}} \sqrt{S}=14 \mathrm{TeV}$ \\
\hline NLO: ABM11 & $\begin{array}{c}7.13_{-0.43}^{+0.0}{ }_{-0.13}^{+0.13} \\
\left(7.25_{-0.48}^{+0.0}{ }_{-0.13}^{+0.13}\right)\end{array}$ & $\begin{array}{c}139.5_{-13.4}^{+7.4}{ }_{-6.7}^{+6.7} \\
\left(145.4_{-14.7}^{+8.2}{ }_{-6.3}^{+6.3}\right)\end{array}$ & $\begin{array}{c}204.0_{-19.6-9.0}^{+11.6+9.0} \\
\left(212.6_{-21.5}^{+12.9}+8.5\right)\end{array}$ & $\begin{array}{c}853.9_{-77.9}^{+59.2+26.6} \\
\left(896.3_{-86.7}^{+66.7}+26.8\right)\end{array}$ \\
\hline CT10 & $\begin{array}{c}7.57_{-0.48}^{+0.0}{ }_{-0.35}^{+0.48} \\
\left(7.66_{-0.49}^{+0.0}{ }_{-0.37}^{+0.53}\right)\end{array}$ & $\begin{array}{c}175.1_{-17.9}^{+10.9}+14.7 \\
\left(174.1_{-17 .{ }_{-1}^{+10.9}+14.2}^{+17.5}\right)\end{array}$ & $\begin{array}{c}250.0_{-25.2}^{+16.5}+18.5 \\
\left(248.6_{-25.0}^{+16.4}+18.1\right)\end{array}$ & $\begin{array}{c}967.4_{-90.8}^{+74.9}-_{-36.8}^{+41.5} \\
\left(964.6_{-90.9}^{+74.6}-_{-40.1}^{+43.3}\right)\end{array}$ \\
\hline NNLO: ABM11 & $7.22_{-0.10}^{+0.10+0.16}+0.16$ & $143.8_{-4.3}^{+0.2+6.4}$ & $210.4_{-6.3-8.6}^{+0.1+8.6}$ & $880.0_{-24.0}^{+0.0}+24.6$ \\
\hline CT10 & $7.70_{-0.15}^{+0.10+0.47}+0.35$ & $180.7_{-5.8}^{+0.0}+11.1$ & $258.0_{-8.1}^{+0.0}{ }_{-14.1}^{+17.2}$ & $997.9_{-28.3}^{+0.0}+38.1$ \\
\hline
\end{tabular}

the mass in the $\overline{\mathrm{MS}}$ scheme, we briefly recall the kinematics of heavy-quark hadro-production,

$h_{1}\left(P_{1}\right)+h_{2}\left(P_{2}\right) \longrightarrow \mathrm{Q}\left(p_{1}\right)+X[\overline{\mathrm{Q}}]\left(p_{X}\right)$,

where $h_{1}$ and $h_{2}$ are hadrons, $X[\overline{\mathrm{Q}}]$ denotes any allowed hadronic final state containing at least the heavy anti-quark, and $\mathrm{Q}\left(p_{1}\right)$ is the identified heavy-quark with mass $m$. The hadronic invariants in this reaction are

$S=\left(P_{1}+P_{2}\right)^{2}, \quad T_{1}=\left(P_{2}-p_{1}\right)^{2}-m^{2}$,

$U_{1}=\left(P_{1}-p_{1}\right)^{2}-m^{2}$.

The double differential cross section for Eq. (5) in terms of the hard parton cross section $\sigma_{i j}$ and PDFs $f_{i}$ at the factorization scale $\mu^{2}$ reads

$$
\begin{aligned}
S^{2} \frac{d^{2} \sigma\left(S, T_{1}, U_{1}\right)}{\mathrm{d} T_{1} \mathrm{~d} U_{1}}= & \int_{x_{1}^{-}}^{1} \frac{d x_{1}}{x_{1}} \int_{x_{2}^{-}}^{1} \frac{\mathrm{d} x_{2}}{x_{2}} f_{i}\left(x_{1}, \mu^{2}\right) f_{j}\left(x_{2}, \mu^{2}\right) \\
& \times s^{2} \frac{d^{2} \sigma_{i j}\left(s, t_{1}, u_{1}, \mu^{2}\right)}{\mathrm{d} t_{1} \mathrm{~d} u_{1}}
\end{aligned}
$$

and the partonic invariants are related to their hadronic counterparts through

$t_{1}=x_{1} T_{1}, \quad u_{1}=x_{2} U_{1}, \quad s=x_{1} x_{2} S$,

with the limits on $x_{1}$ and $x_{2}$,

$x_{1}^{-}=-\frac{U_{1}}{S+T_{1}} \leq x_{1} \leq 1, \quad x_{2}^{-}=\frac{x_{1} T_{1}}{x_{1} S+U_{1}} \leq x_{2} \leq 1$.

In order to write the differential cross section in terms of $p_{\mathrm{T}}^{t}, y^{t}$ and $m^{t \bar{t}}$, we will also need their definitions in terms of the hadronic invariants. For the case of $p_{\mathrm{T}}^{t}$ and $y^{t}$, the relations are

$y^{t}=\frac{1}{2} \ln \left(\frac{T_{1}}{U_{1}}\right), \quad\left(p_{\mathrm{T}}^{t}\right)^{2}=\frac{T_{1} U_{1}}{S}-m^{2}$,

whereas for $m^{t \bar{t}}$, pair-invariant mass kinematics is used, in which case the requirements on the integrals are

$x_{1}^{-}=\frac{\left(m^{t \bar{t}}\right)^{2}}{S}$ and $x_{2}^{-}=\frac{\left(m^{t \bar{t}}\right)^{2}}{x_{1} S}$.
In these kinematics, the relevant partonic invariants for writing the differential cross section in terms of $m^{t \bar{t}}$ are,

$t_{1}=-\frac{\left(m^{t \bar{t}}\right)^{2}}{2}\left(1-\beta_{t} \cos \theta\right)$,
$u_{1}=-\frac{\left(m^{t \bar{t}}\right)^{2}}{2}\left(1+\beta_{t} \cos \theta\right)$,

with $\beta_{t}=\sqrt{1-4 m^{2} /\left(m^{t \bar{t}}\right)^{2}}$ and $\theta$ the scattering angle of the top quark. Full discussions of the kinematics to NLO for one-particle inclusive and pair-invariant mass kinematics are available in $[11,38]$ respectively.

In order to convert to cross section predictions with the mass in the $\overline{\mathrm{MS}}$ scheme, we start from the on-shell description:

$$
\begin{aligned}
\frac{\mathrm{d} \sigma\left(m_{t}^{\text {pole }}\right)}{\mathrm{d} X}= & \left(\frac{\alpha_{\mathrm{s}}}{\pi}\right)^{2} \frac{\mathrm{d} \sigma^{(0)}\left(m_{t}^{\text {pole }}\right)}{\mathrm{d} X} \\
& +\left(\frac{\alpha_{\mathrm{s}}}{\pi}\right)^{3} \frac{\mathrm{d} \sigma^{(1)}\left(m_{t}^{\text {pole }}\right)}{\mathrm{d} X}+\mathscr{O}\left(\alpha_{\mathrm{s}}^{4}\right)
\end{aligned}
$$

where $X$ denotes any of the variables $p_{\mathrm{T}}^{t}, y^{t}$ and so on. If we now replace $m_{t}^{\text {pole }}$ with $m\left(\mu_{\mathrm{r}}\right)$ using Eq. (1), we can expand in $\alpha_{\mathrm{s}}$ and obtain a description of the differential cross section in the $\overline{\mathrm{MS}}$ scheme.

$$
\begin{aligned}
\frac{\mathrm{d} \sigma\left(m\left(\mu_{\mathrm{r}}\right)\right)}{\mathrm{d} X}= & \left(\frac{\alpha_{\mathrm{s}}}{\pi}\right)^{2} \frac{\mathrm{d} \sigma^{(0)}\left(m\left(\mu_{\mathrm{r}}\right)\right)}{\mathrm{d} X} \\
& +\left(\frac{\alpha_{\mathrm{s}}}{\pi}\right)^{3}\left\{\frac{\mathrm{d} \sigma^{(1)}\left(m\left(\mu_{\mathrm{r}}\right)\right)}{\mathrm{d} X}+d_{1} m\left(\mu_{\mathrm{r}}\right)\right. \\
& \left.\times\left.\frac{\mathrm{d}}{\mathrm{d} m_{t}}\left(\frac{\mathrm{d} \sigma^{(0)}\left(m_{t}\right)}{\mathrm{d} X}\right)\right|_{m_{t}=m\left(\mu_{\mathrm{r}}\right)}\right\}+\mathscr{O}\left(\alpha_{\mathrm{s}}^{4}\right) .
\end{aligned}
$$

The only extra part required is the mass derivative of the Born contribution. This has been computed semi-analytically for the $p_{\mathrm{T}}^{t}, y^{t}$, and $m^{t \bar{t}}$ distributions. To see why we also need some numerical derivatives in this calculation, consider Eq. (6) for the Born contribution to the double differential cross section as a starting point: 


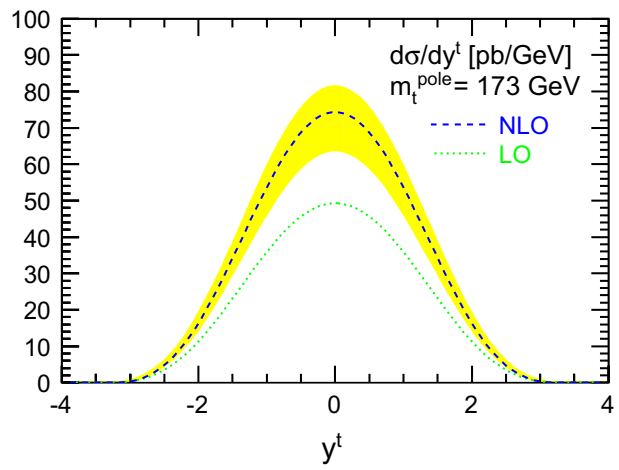

Fig. 4 The differential cross section with respect to the rapidity $y^{t}$ of the top quark in the pole (left) and the $\overline{\mathrm{MS}}$ (right) mass scheme at the LHC with $\sqrt{S}=8 \mathrm{TeV}$. The dotted (green) curves are the LO contributions while the dashed (blue) curves include NLO corrections and

$$
\begin{aligned}
& \int_{x_{1}^{-}}^{1} \frac{\mathrm{d} x_{1}}{x_{1}} \int_{x_{2}^{-}}^{1} \frac{\mathrm{d} x_{2}}{x_{2}} f_{i}\left(x_{1}, \mu^{2}\right) f_{j}\left(x_{2}, \mu^{2}\right) s^{2} \\
& \times \frac{\mathrm{d}^{2} \sigma_{i j}^{(0)}}{\mathrm{d} t_{1} \mathrm{~d} u_{1}} \delta\left(s+t_{1}+u_{1}\right),
\end{aligned}
$$

where the delta function imposes Born kinematics and can be used to carry out the integral over $x_{2}$ through its relation to $s, t_{1}$ and $u_{1}$. Re-writing the cross section in terms of $p_{\mathrm{T}}^{t}$ and $y^{t}$ provides us with the form of the integrand that will need to be evaluated,

$$
\left.\int_{x_{1}^{-}}^{1} \mathrm{~d} x_{1} \mathscr{L}\left(x_{1}, x_{2}, \mu^{2}\right) \frac{x_{1} x_{2} S}{x_{1} S+U_{1}} \frac{\mathrm{d}^{2} \sigma\left(s, t_{1}, u_{1}\right)}{\mathrm{d} y^{t} \mathrm{~d} p_{\mathrm{T}}^{t 2}}\right|_{x_{2}=-\frac{x_{1} T_{1}}{x_{1} S+U_{1}}},
$$

where $\mathscr{L}\left(x_{1}, x_{2}, \mu^{2}\right)=f_{1}\left(x_{1}, \mu^{2}\right) f_{2}\left(x_{2}, \mu^{2}\right) / x_{1} x_{2}$ is the differential parton luminosity.

The most important aspect to note is that both $x_{2}$ and $x_{1}^{-}$ depend on the top-quark mass through their relations to the Mandelstam variables. This means that the mass derivative of the PDFs needs to be done numerically using

$$
\begin{aligned}
\frac{\mathrm{d}}{\mathrm{d} m} & \mathscr{L}\left(x_{1}, x_{2}, \mu^{2}\right) \\
= & \frac{\mathrm{d} x_{1}}{\mathrm{~d} m} \frac{\mathscr{L}\left(x_{1}+\delta, x_{2}, \mu^{2}\right)-\mathscr{L}\left(x_{1}-\delta, x_{2}, \mu^{2}\right)}{2 \delta} \\
& +\frac{\mathrm{d} x_{2}}{\mathrm{~d} m} \frac{\mathscr{L}\left(x_{1}, x_{2}+\delta, \mu^{2}\right)-\mathscr{L}\left(x_{1}, x_{2}-\delta, \mu^{2}\right)}{2 \delta} .
\end{aligned}
$$

This form of the derivative is found to converge well. Aside from this, all other derivatives are known analytically. When compared with a fully numerical calculation of the derivative term, it is found that the two methods agree to less than $1 \%$. In the case of $m^{t \bar{t}}$, the integration limits and variables do not depend on the top-quark mass $(m)$ so all derivatives are computed analytically.

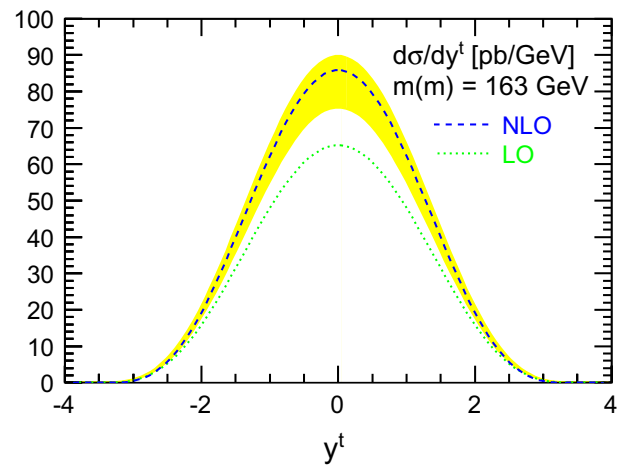

are obtained using the PDF set CT10 [36]. The scale dependence in the range $\mu / m_{t}^{\text {pole }}$ or $\mu / m(m) \in[1 / 2,2]$ is shown as a band around the NLO curve

Using the relations presented here, we have computed the differential cross sections for $t \bar{t}$-production in terms of $p_{\mathrm{T}}^{t}, y^{t}$ and $m^{t \bar{t}}$. We have used the program MCFM [39] for the NLO corrections $[13,40]$ in the conventional pole mass $m_{t}^{\text {pole }}$ scheme and a custom routine for the Born and mass derivative terms. The calculations were carried out using the ABM11 [30] and CT10 [36] PDFs at NLO. As a check, each curve was integrated to obtain a result for the full cross section. In all cases, the value agreed within less than $1 \%$ of the cross section computed using Hathor. As well, the mass derivatives were checked by computing the differential cross sections at values of the top mass ranging between 150 and $180 \mathrm{GeV}$. A curve was fit to each point in the relevant spectrum to obtain the derivative at the given $\overline{\mathrm{MS}}$ mass. Again, these values agreed within less than $1 \%$ of the (semi-)analytic derivatives used.

In Fig. 4 the rapidity distributions are shown for the $\overline{\mathrm{MS}}$ and pole mass schemes. It is clear from these that at NLO, the convergence of the perturbative series as well as the scale dependence improves. In the pole-mass scheme, a relative increase for the cross section ratios $\sigma_{\mathrm{NLO}} / \sigma_{\mathrm{LO}}=1.50$ is seen, while in the $\overline{\mathrm{MS}}$ scheme we have $\sigma_{\mathrm{NLO}} / \sigma_{\mathrm{LO}}=1.31$ at $y^{t}=0$. The scale variation in the on-shell scheme is $\Delta \sigma_{\mathrm{NLO}}={ }_{-14 \%}^{+9.5 \%}$ while in the $\overline{\mathrm{MS}}$ scheme, we have $\Delta \sigma_{\mathrm{NLO}}=$ ${ }_{-12 \%}^{+4.5 \%}$ again at $y^{t}=0$.

Figure 5 shows the transverse momentum distributions. Again we see an improvement when moving from the pole mass scheme to the $\overline{\mathrm{MS}}$ scheme. In this case the improvement in the NLO contribution is a bit better with $\sigma_{\mathrm{NLO}} / \sigma_{\mathrm{LO}}=1.50$ for the pole mass scheme and $\sigma_{\mathrm{NLO}} / \sigma_{\mathrm{LO}}=1.25$ in the $\overline{\mathrm{MS}}$ scheme. The scale variation goes from $\Delta \sigma_{\mathrm{NLO}}={ }_{-13 \%}^{+13 \%}$ in the pole mass scheme to $\Delta \sigma_{\mathrm{NLO}}={ }_{-9.6 \%}^{+6.4 \%}$ in the $\overline{\mathrm{MS}}$ scheme. The above values are taken near the maximum of the curve at $p_{\mathrm{T}}^{t}=75 \mathrm{GeV}$.

Finally, in Fig. 6 we show the invariant mass distributions. The increase at NLO here is $\sigma_{\mathrm{NLO}} / \sigma_{\mathrm{LO}}=1.54$ with 

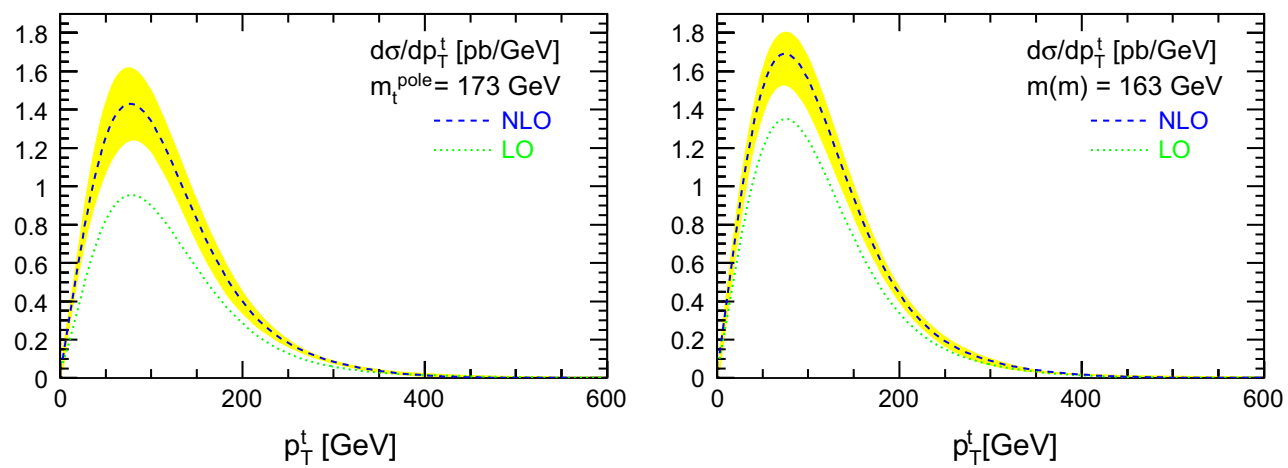

Fig. 5 Same as Fig. 4 for the differential cross section with respect to the transverse momentum $p_{\mathrm{T}}^{t}$ of the top quark
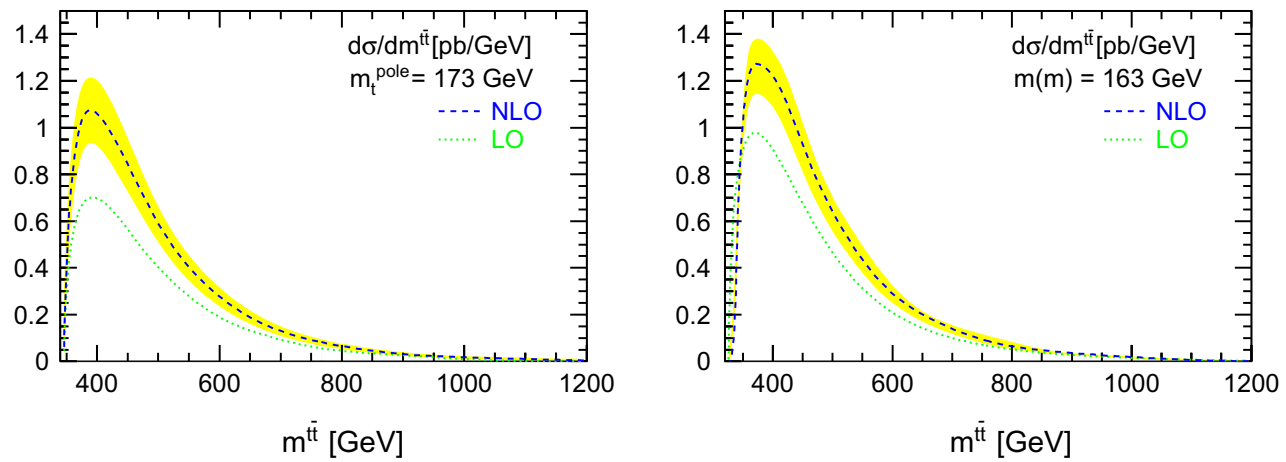

Fig. 6 Same as Fig. 4 for the differential cross section with respect to the invariant mass $m^{t \bar{t}}$ of the top quark pair

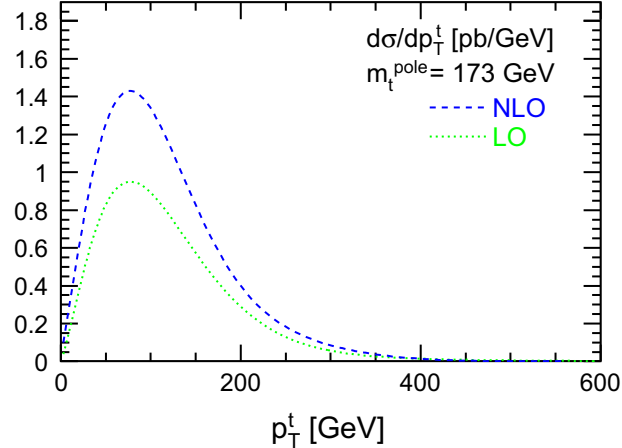

Fig. 7 The differential cross section with respect to the rapidity $p_{\mathrm{T}}^{t}$ of the top quark in the pole (left) and the $\overline{\mathrm{MS}}$ (right) mass scheme at the LHC with $\sqrt{S}=8 \mathrm{TeV}$. The dotted (green) curves are the LO contribu-

scale variation $\Delta \sigma_{\mathrm{NLO}}={ }_{-13 \%}^{+13 \%}$ in the pole mass scheme and $\sigma_{\mathrm{NLO}} / \sigma_{\mathrm{LO}}=1.30$ with scale variation $\Delta \sigma_{\mathrm{NLO}}={ }_{-9.6 \%}^{+8.2 \%}$ in the $\overline{\mathrm{MS}}$ scheme. These values are taken at an invariant mass of $m^{t \bar{t}}=371 \mathrm{GeV}$.

Looking at just the leading order curves in Fig. 5 we see that the change to the $\overline{\mathrm{MS}}$ scheme seems to push the peak to higher values. To be sure that this does not contribute to the better convergence that we are seeing in the $\overline{\mathrm{MS}}$ scheme, we carry out the same analysis except with an $\overline{\mathrm{MS}}$ mass of 173 $\mathrm{GeV}$. Figure 7 shows the LO and NLO contributions in the pole mass scheme and the $\overline{\mathrm{MS}}$ scheme.

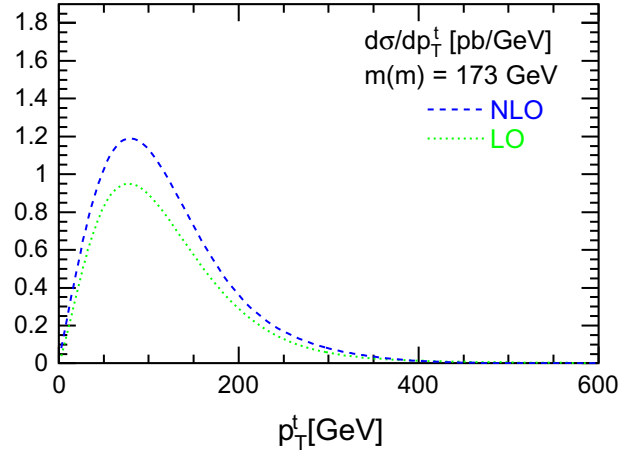

tions while the dashed (blue) curves include NLO corrections and are obtained using the PDF set CT10 [36]

Inspection of the $\mathrm{K}$-factors in these plots shows an increase at $\mathrm{NLO}$ of $\sigma_{\mathrm{NLO}} / \sigma_{\mathrm{LO}}=1.50$ in the pole-mass scheme and $\sigma_{\mathrm{NLO}} / \sigma_{\mathrm{LO}}=1.26$ in the $\overline{\mathrm{MS}}$ scheme. As in the previous discussion, this value is taken at $p_{\mathrm{T}}^{t}=75 \mathrm{GeV}$. This should be compared the the value of $\sigma_{\mathrm{NLO}} / \sigma_{\mathrm{LO}}=1.25$ in the $\overline{\mathrm{MS}}$ scheme using a top mass of $163 \mathrm{GeV}$. We see that the improvement in convergence comes only from the inclusion of the derivative term in Eq. (14) and is largely unaffected by the choice of top mass. Note that this comparison between the two mass choices is consistent through the whole $p_{\mathrm{T}}^{t}$ spectrum and is not a special case at the chosen value of $p_{\mathrm{T}}^{t}$. 


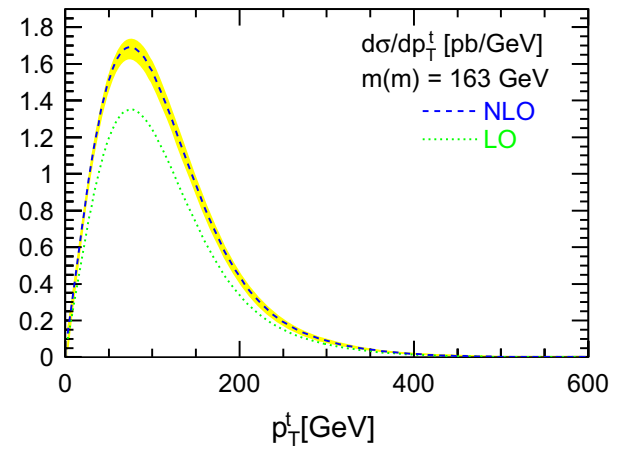

Fig. 8 The scale dependence of the $p_{\mathrm{T}}^{t}$ distribution at NLO using the running mass $m(\mu)$ and the PDF set CT10. The yellow band shows the variation of the differential distribution in the range $\mu / m(m) \in[1 / 2,2]$. This should be compared with the plot in Fig. 5 (right) where the same analysis was carried out using a fixed $m(m)$

As we did for the total cross-section, we also consider the alternative scale choices $m(\mu)$ for the differential crosssections. Figure 8 is the same as Fig. 5 (right) except the band is now produced by using $m(\mu)$ and we vary $\mu / m(m) \in$ $[1 / 2,2]$. Comparing Fig. 5 (right) and Fig. 8 we see that using a fixed $m(m)$ is the more conservative choice when considering scale dependence at NLO. We now have $\Delta \sigma_{\mathrm{NLO}}={ }_{-3.7 \%}^{+2.3 \%}$ at $p_{\mathrm{T}}^{t}=75 \mathrm{GeV}$. This improvement in the scale dependence can also be seen in the NLO total cross-section in Fig. 3.

Let us also comment on the difference between the size of predictions of the $\overline{\mathrm{MS}}$ scheme and pole scheme at NLO. When comparing, for example, the peak height in the $p_{\mathrm{T}}^{t}$ distributions in Fig. 5 it is seen that the height differs by approximately $15 \%$. This difference can be attributed to the fact that we are using fixed order perturbation theory and reinforces the observation of faster convergence in the $\overline{\mathrm{MS}}$ scheme. The same effect can be seen at NLO in the total crosssection in Fig. 1, where the difference is again approximately $15 \%$. At NNLO however, the cross-section predictions are somewhat closer and only differ by about $6 \%$. A similar 'convergence' of the predicted distributions is expected when higher order corrections are included. Given that the NNLO contribution to the total cross-section is approximately $12 \%$ in the pole mass scheme and only $3 \%$ in the $\overline{\mathrm{MS}}$ scheme, we expect most of this difference to be made up by higher order corrections that are larger in the pole mass scheme than in the $\overline{\mathrm{MS}}$ scheme.

In addition to these improvements, moving from the pole mass to the $\overline{\mathrm{MS}}$ scheme changes the overall shape of the distributions so that the peaks generally become more pronounced. This can be attributed to our choice of mass and is a consequence of the radiative corrections being shifted to the threshold region as mentioned earlier. However, the peak positions in both the $p_{\mathrm{T}}^{t}$ and $m^{t \bar{t}}$ distributions are stable against radiative corrections. At most they are seen to shift by $1 \%$, which is unlike the case for $t \bar{t}$-production from $e^{+} e^{-}$ collisions where the position of the $t \bar{t}$-threshold peak shifts significantly upon adding NLO and NNLO perturbative corrections to the total cross section expressed in terms of the pole mass [41].

Another salient feature not shown in Fig. 6 occurs in the $\overline{\mathrm{MS}}$ differential cross section with respect to the invariant mass of the $t \bar{t}$ pair. Very close to the threshold of $t \bar{t}$ production the contribution reponsible for the change in the mass renormalization scheme, i.e., the derivative term in Eq. (14), becomes large. This is due to the presence of a $1 / \beta_{t}$ which diverges as $m^{t \bar{t}} \rightarrow m$, cf. Eq. (11). These large corrections have the effect of causing the invariant mass spectrum to dip below zero for values of $m^{t \bar{t}} \gtrsim 2 m_{t}$. The area under this (negative) divergent part of the curve represents approximately $-0.7 \%$ of the total area (i.e., total cross-section). In the full spectrum, however, this is counterbalanced by the positive contribution resulting in a cross section integrated over $m^{t \bar{t}}$ that agrees within less than $1 \%$ with the value calculated in Hathor.

Obviously, this behavior is an indication of the breakdown of fixed-order perturbation theory. First of all, bound-state effects in $t \bar{t}$ production at hadron colliders arise in the kinematic region $m^{t \bar{t}} \gtrsim 2 m_{t}$, i.e., when the velocity $\beta$ of the top quarks is small, $\beta \ll 1$. In this region, the conventional perturbative expansion in $\alpha_{\mathrm{s}}$ breaks down, owing to singular terms $\sim\left(\alpha_{\mathrm{s}} / \beta\right)^{n}$ in the $n$-loop amplitude, which require the all-order resummation of the Coulomb corrections [42,43]. This resummation for $t \bar{t}$ dynamics close to threshold is carried out in a non-relativistic effective theory by means of a Schrödinger equation for which the pole mass definition seems to be the natural choice and which implies a certain power counting, so that all terms of order $m_{t} \beta^{2} \sim m_{t} \alpha_{\mathrm{s}}^{2}$ are formally of equal size.

If the contribution for the change in the mass renormalization scheme $\delta m^{\text {sd }}$ from the pole mass to a so-called shortdistance mass $m_{t}^{\text {sd }}$ such as the $\overline{\mathrm{MS}}$ mass $m\left(\mu_{\mathrm{r}}\right)$ is parametrically larger than $m_{t} \alpha_{\mathrm{s}}^{2}$ that is $\delta m^{\text {sd }} \equiv m_{t}^{\text {pole }}-m^{\text {sd }} \sim m_{t}^{\text {sd }} \alpha_{\mathrm{s}}$, then $\delta m^{\text {sd }}$ becomes the dominant term in the kinematic region $m^{t \bar{t}} \gtrsim 2 m_{t}$. Such situation is realized for $\delta m^{\text {sd }} \sim m_{t} \alpha_{\mathrm{s}}$, cf. Eq. (14), and excludes the $\overline{\mathrm{MS}}$ mass from being a useful mass near threshold. Of course, all these findings on the scheme choice for the mass definition close to the threshold are long known from studies for $t \bar{t}$ production in $e^{+} e^{-}$collisions [41] (see $[44,45]$ for recent progress). Various solutions have been proposed, e.g., the alternative use of a so-called $1 S$ mass $[46,47]$ defined through the perturbative contribution to the mass of a hypothetical $n=1,{ }^{3} S_{1}$ toponium bound state, cf. [37] for an application to $t \bar{t}$ hadro-production or the use of a "potential-subtracted" (PS) mass [48], recently considered in [49] in the context of finite-width effects in unstable-particle production at hadron colliders. In any case, since the conventional perturbative expansion of the cross section breaks down for $m^{t \bar{t}} \gtrsim 2 m_{t}$ we do not display this particular kinematic region in Fig. 6. Moreover, with the cur- 
Table 3 Values for the $y^{t}$ differential cross section for top-quark pairproduction at LO and NLO for various $y^{t}$ using the PDF set CT10 [36] with $\sqrt{S}=8 \mathrm{TeV}$. All rates are in $\mathrm{pb}$

\begin{tabular}{llllll}
\hline $\mathrm{d} \sigma$ & \multicolumn{2}{l}{$m_{t}^{\text {pole }}$} & & & \multicolumn{2}{l}{$m(m)$} & \\
\cline { 2 - 3 } \cline { 5 - 6 } & LO & NLO & & LO & NLO \\
\hline$y^{t}=0.2$ & 48.70 & 73.43 & & 64.46 & 84.83 \\
$y^{t}=0.6$ & 44.12 & 66.34 & & 58.57 & 76.74 \\
$y^{t}=1.0$ & 35.90 & 53.70 & & 48.00 & 62.29 \\
$y^{t}=1.4$ & 25.77 & 38.19 & & 34.87 & 44.51 \\
$y^{t}=2.0$ & 11.37 & 16.39 & & 15.93 & 19.34 \\
\hline
\end{tabular}

Table 4 The same as Table 3 but using the PDF set ABM11 [30]

\begin{tabular}{|c|c|c|c|c|}
\hline \multirow[t]{2}{*}{$\frac{\mathrm{d} \sigma}{\mathrm{d} y^{t}}$} & \multicolumn{2}{|l|}{$m_{t}^{\text {pole }}$} & \multicolumn{2}{|l|}{$m(m)$} \\
\hline & $\mathrm{LO}$ & NLO & LO & NLO \\
\hline$y^{t}=0.2$ & 44.39 & 65.82 & 59.51 & 76.33 \\
\hline$y^{t}=0.6$ & 39.55 & 58.57 & 53.18 & 68.00 \\
\hline$y^{t}=1.0$ & 31.07 & 45.89 & 42.06 & 53.44 \\
\hline$y^{t}=1.4$ & 21.04 & 30.91 & 28.83 & 36.18 \\
\hline$y^{t}=2.0$ & 8.018 & 11.55 & 11.40 & 13.72 \\
\hline
\end{tabular}

Table 5 Values for the $p_{\mathrm{T}}^{t}$ differential cross section for top-quark pairproduction at LO and NLO for various $p_{\mathrm{T}}^{t}$ using the PDF set CT10 [36]. All rates are in $\mathrm{pb} / \mathrm{GeV}$

\begin{tabular}{|c|c|c|c|c|}
\hline \multirow[t]{2}{*}{$\frac{\mathrm{d} \sigma}{\mathrm{d} p_{\mathrm{T}}^{t}}$} & \multicolumn{2}{|l|}{$m_{t}^{\text {pole }}$} & \multicolumn{2}{|l|}{$m(m)$} \\
\hline & LO & NLO & LO & NLO \\
\hline$p_{\mathrm{T}}^{t}=30 \mathrm{GeV}$ & 0.5513 & 0.8681 & 0.8214 & 1.058 \\
\hline$p_{\mathrm{T}}^{t}=90 \mathrm{GeV}$ & 0.9364 & 1.399 & 1.308 & 1.637 \\
\hline$p_{\mathrm{T}}^{t}=130 \mathrm{GeV}$ & 0.7130 & 1.045 & 0.9419 & 1.196 \\
\hline$p_{\mathrm{T}}^{t}=170 \mathrm{GeV}$ & 0.4422 & 0.6288 & 0.5455 & 0.7057 \\
\hline$p_{\mathrm{T}}^{t}=230 \mathrm{GeV}$ & 0.1777 & 0.2496 & 0.2070 & 0.2675 \\
\hline$p_{\mathrm{T}}^{t}=290 \mathrm{GeV}$ & 0.06806 & 0.09941 & 0.08152 & 0.1035 \\
\hline$p_{\mathrm{T}}^{t}=360 \mathrm{GeV}$ & 0.02533 & 0.03105 & 0.02756 & 0.03537 \\
\hline
\end{tabular}

rently given experimental resolution for the $m^{t \bar{t}}$-bins, cf. [4], it will be difficult to access this region at the LHC at all.

For completeness we also provide a table of values for the cross section at LHC with $\sqrt{S}=8 \mathrm{TeV}$ at binned values of $y^{t}, p_{\mathrm{T}}^{t}$ and $m^{t \bar{t}}$ with binning approximately equal to that of [4]. Comparing the data generated using ABM11 as compared to CT10, we see that there is an overall shift downward consistent with that observed for the total cross section, cf. Tables 1 and 2. The improvement of the apparent perturbative convergence and the scale stability when moving from the pole mass scheme to the $\overline{\mathrm{MS}}$ scheme is consistent for both PDF sets (Table 4).
Table 6 The same as Table 5 but using the PDF set ABM11 [30]

\begin{tabular}{|c|c|c|c|c|}
\hline \multirow[t]{2}{*}{$\frac{\mathrm{d} \sigma}{\mathrm{d} p_{\mathrm{T}}^{t}}$} & \multicolumn{2}{|l|}{$m_{t}^{\text {pole }}$} & \multicolumn{2}{|l|}{$m(m)$} \\
\hline & LO & NLO & LO & NLO \\
\hline$p_{\mathrm{T}}^{t}=30 \mathrm{GeV}$ & 0.4874 & 0.7568 & 0.7467 & 0.9220 \\
\hline$p_{\mathrm{T}}^{t}=90 \mathrm{GeV}$ & 0.8141 & 1.206 & 1.148 & 1.429 \\
\hline$p_{\mathrm{T}}^{t}=130 \mathrm{GeV}$ & 0.6076 & 0.8862 & 0.8053 & 1.006 \\
\hline$p_{\mathrm{T}}^{t}=170 \mathrm{GeV}$ & 0.3658 & 0.5262 & 0.4429 & 0.5843 \\
\hline$p_{\mathrm{T}}^{t}=230 \mathrm{GeV}$ & 0.1425 & 0.1954 & 0.1750 & 0.2175 \\
\hline$p_{\mathrm{T}}^{t}=290 \mathrm{GeV}$ & 0.05567 & 0.06975 & 0.06227 & 0.0731 \\
\hline$p_{\mathrm{T}}^{t}=360 \mathrm{GeV}$ & 0.02008 & 0.02415 & 0.01266 & 0.0181 \\
\hline
\end{tabular}

Table 7 Values for the $m^{t \bar{t}}$ differential cross section for top-quark pairproduction at LO and NLO for various $m^{t \bar{t}}$ using the PDF set CT10 [36]. All rates are in $\mathrm{pb} / \mathrm{GeV}$

\begin{tabular}{|c|c|c|c|c|}
\hline \multirow[t]{2}{*}{$\frac{\mathrm{d} \sigma}{\mathrm{d} m^{t t}}$} & \multicolumn{2}{|l|}{$m_{t}^{\text {pole }}$} & \multicolumn{2}{|l|}{$m(m)$} \\
\hline & $\mathrm{LO}$ & NLO & $\mathrm{LO}$ & NLO \\
\hline$m^{t \bar{t}}=350 \mathrm{GeV}$ & 0.2985 & 0.4278 & 0.9046 & 1.0295 \\
\hline$m^{t \bar{t}}=450 \mathrm{GeV}$ & 0.5648 & 0.8441 & 0.6755 & 0.9270 \\
\hline$m^{t \bar{t}}=500 \mathrm{GeV}$ & 0.4022 & 0.5914 & 0.4656 & 0.6403 \\
\hline$m^{t \bar{t}}=600 \mathrm{GeV}$ & 0.1898 & 0.2782 & 0.2102 & 0.2917 \\
\hline$m^{t \bar{t}}=700 \mathrm{GeV}$ & 0.09342 & 0.1301 & 0.09977 & 0.1404 \\
\hline$m^{t \bar{t}}=950 \mathrm{GeV}$ & 0.01796 & 0.02343 & 0.02067 & 0.02740 \\
\hline
\end{tabular}

Table 8 The same as Table 7 but using the PDF set ABM11 [30]

\begin{tabular}{llllll}
\hline$\frac{\mathrm{d} \sigma}{\mathrm{d} m^{t \bar{t}}}$ & $m_{t}^{\text {pole }}$ & & & $m(m)$ & \\
\cline { 2 - 3 } \cline { 5 - 6 } & LO & NLO & & LO & NLO \\
\hline$m^{t \bar{t}}=350 \mathrm{GeV}$ & 0.3036 & 0.4546 & & 0.8420 & 0.9508 \\
$m^{t \bar{t}}=450 \mathrm{GeV}$ & 0.4967 & 0.7381 & & 0.5914 & 0.8103 \\
$m^{t \bar{t}}=500 \mathrm{GeV}$ & 0.3481 & 0.5118 & & 0.3964 & 0.54488 \\
$m^{t \bar{t}}=600 \mathrm{GeV}$ & 0.1554 & 0.2212 & & 0.1704 & 0.2357 \\
$m^{t \bar{t}}=700 \mathrm{GeV}$ & 0.0729 & 0.09674 & 0.07706 & 0.1061 \\
$m^{t \bar{t}}=950 \mathrm{GeV}$ & 0.01326 & 0.01839 & 0.01407 & 0.01611
\end{tabular}

In summary, we have shown how treating the differential cross sections for $t \bar{t}$ production in the $\overline{\mathrm{MS}}$ scheme for the topquark mass has benefits as compared to the pole mass scheme. The perturbative series shows the same improvement in convergence and scale dependence as has been observed for the total cross section. As a consequence the NLO contributions with a $\overline{\mathrm{MS}}$ mass are expected to provide already very precise cross section predictions. An extension to NNLO accuracy would provide results with a still smaller theoretical uncertainty from the scale variation. Yet, the predictions at the nominal scale, i.e., $\mu_{\mathrm{r}}=m(m)$, are expected to remain largely unchanged (Tables 6, 8). 
As future prospects we note that the refinement of the present phenomenological analysis to NNLO accuracy is certainly feasible once the complete NNLO QCD corrections for differential $t \bar{t}$ production are available. As a first step in this direction, one may consider approximate NNLO corrections based, e.g., on the dominant threshold logarithms. Other obvious improvements are extension to double-differential distributions and other exclusive observables, even including top-quark decay.

Acknowledgments This work is partially supported by the Deutsche Forschungsgemeinschaft in Sonderforschungsbereich/Transregio 9 and by the European Commission through contract PITN-GA-2010-264564 (LHCPhenoNet).

Open Access This article is distributed under the terms of the Creative Commons Attribution License which permits any use, distribution, and reproduction in any medium, provided the original author(s) and the source are credited.

Funded by $\mathrm{SCOAP}^{3}$ / License Version CC BY 4.0.

\section{References}

1. CMS Collaboration, CMS-PAS-TOP-12-003 (2012)

2. ATLAS Collaboration, ATLAS-CONF-2012-149, ATLAS-COMCONF-2012-170 (2012)

3. CMS Collaboration, CMS-PAS-TOP-12-006 (2012)

4. CMS Collaboration, S. Chatrchyan et al., Eur. Phys. J. C73, 2339 (2013). arXiv:1211.2220

5. ATLAS Collaboration, G. Aad et al., Eur. Phys. J. C73, 2261 (2013). arXiv: 1207.5644

6. P. Bärnreuther, M. Czakon, A. Mitov, Phys. Rev. Lett. 109, 132001 (2012). arXiv:1204.5201

7. M. Czakon, A. Mitov, JHEP 1212, 054 (2012). arXiv: 1207.0236

8. M. Czakon, A. Mitov, JHEP 1301, 080 (2013). arXiv:1210.6832

9. M. Czakon, P. Fiedler, A. Mitov, Phys. Rev. Lett. 110, 252004 (2013). arXiv:1303.6254

10. W. Beenakker, H. Kuijf, W. van Neerven, J. Smith, Phys. Rev. D 40, 54 (1989)

11. M.L. Mangano, P. Nason, G. Ridolfi, Nucl. Phys. B 373, 295 (1992)

12. K. Melnikov, M. Schulze, JHEP 0908, 049 (2009). arXiv:0907.3090

13. J.M. Campbell, R.K. Ellis, (2012). arXiv:1204.1513

14. G. Bevilacqua et al., JHEP 1102, 083 (2011). arXiv:1012.4230

15. A. Denner et al., Phys. Rev. Lett. 106, 052001 (2011). arXiv: 1012.3975

16. A. Denner et al., JHEP 1210, 110 (2012). arXiv: 1207.5018

17. N. Kidonakis, Phys. Rev. D 82, 114030 (2010). arXiv:1009.4935

18. V. Ahrens et al., Phys. Lett. B 687, 331 (2010). arXiv:0912.3375
19. M. Guzzi, K. Lipka, S. Moch, arXiv: 1406.0386

20. A. Broggio, A.S. Papanastasiou, A. Signer, arXiv:1407.2532

21. F. Bezrukov, M.Y. Kalmykov, B.A. Kniehl, M. Shaposhnikov, JHEP 1210, 140 (2012). arXiv: 1205.2893

22. S. Alekhin, A. Djouadi, S. Moch, Phys. Lett. B 716, 214 (2012). arXiv: 1207.0980

23. U. Langenfeld, S. Moch, P. Uwer, Phys. Rev. D 80, 054009 (2009). arXiv:0906.5273

24. S. Alioli et al., Eur. Phys. J. C 73, 2438 (2013). arXiv:1303.6415

25. I.I. Bigi, M.A. Shifman, N. Uraltsev, A. Vainshtein, Phys. Rev. D 50, 2234 (1994). arXiv:hep-ph/9402360

26. M. Beneke, V.M. Braun, Nucl. Phys. B 426, 301 (1994). arXiv:hep-ph/9402364

27. N. Gray, D.J. Broadhurst, W. Grafe, K. Schilcher, Z. Phys. C 48, 673 (1990)

28. K. Chetyrkin, M. Steinhauser, Nucl. Phys. B 573, 617 (2000). arXiv:hep-ph/9911434

29. K. Melnikov, T.v. Ritbergen, Phys. Lett. B 482, 99 (2000). arXiv:hep-ph/9912391

30. S. Alekhin, J. Blümlein, S. Moch, Phys. Rev. D 86, 054009 (2012). arXiv: 1202.2281

31. S. Moch, P. Uwer, A. Vogt, Phys. Lett. B 714, 48 (2012). arXiv: 1203.6282

32. M. Aliev et al., Comput. Phys. Commun. 182, 1034 (2011). arXiv: 1007.1327

33. S. Moch, U. Langenfeld, P. Uwer, PoS RADCOR2009, 030 (2010). arXiv: 1001.3987

34. D0 Collaboration, V.M. Abazov et al., Phys. Lett. B 703, 422 (2011), arXiv:1104.2887

35. S. Alekhin, J. Blümlein, S. Moch, arXiv:1310.3059

36. J. Gao et al., (2013). arXiv:1302.6246

37. V. Ahrens et al., Phys. Lett. B 703, 135 (2011). arXiv:1105.5824

38. W. Beenakker et al., Nucl. Phys. B 351, 507 (1991)

39. J.M. Campbell, R. Ellis, C. Williams. MCFM webpage. http:// mcfm.fnal.gov

40. J.M. Campbell, R. Ellis, Nucl. Phys. Proc. Suppl. 205-206, 10 (2010). arXiv:1007.3492

41. A. Hoang et al., Eur. Phys. J. direct C 2, 1 (2000). arXiv:hep-ph/0001286

42. K. Hagiwara, Y. Sumino, H. Yokoya, Phys. Lett. B 666, 71 (2008). arXiv:0804.1014

43. Y. Kiyo et al., Eur. Phys. J. C 60, 375 (2009). arXiv:0812.0919

44. A.H. Hoang, M. Stahlhofen, JHEP 1405, 121 (2014). arXiv: 1309.6323

45. M. Beneke, Y. Kiyo, K. Schuller, arXiv:1312.4791

46. A.H. Hoang, Z. Ligeti, A.V. Manohar, Phys. Rev. D 59, 074017 (1999). arXiv:hep-ph/9811239

47. A. Hoang, T. Teubner, Phys. Rev. D 60, 114027 (1999). arXiv:hep-ph/9904468

48. M. Beneke, Phys. Lett. B 434, 115 (1998). arXiv:hep-ph/9804241

49. P. Falgari, A. Papanastasiou, A. Signer, JHEP 1305, 156 (2013). arXiv: 1303.5299 